\title{
Cross-contamination: Comparison of Nasal and Chronic Leg Ulcer Staphy- lococcus aureus Strains Isolated from the Same Patient
}

\author{
Kristine Gjødsbøl ${ }^{1,2}$, Mette Elena Skindersoe ${ }^{1}$, Robert Leo Skov ${ }^{1}$ and Karen Angeliki Krogfelt ${ }^{1, *}$ \\ ${ }^{1}$ Microbiology and Infection Control, Statens Serum Institut, Artillerivej 5, DK-2300 Copenhagen S, Denmark \\ ${ }^{2}$ Coloplast A/S, Holtedam 1, DK-3050 Humlebaek, Denmark
}

\begin{abstract}
The aim of this study was to investigate the occurrence of bacterial cross-contamination between the nasal cavity and leg ulcers. Sixteen patients were included in the study. Staphylococcus aureus was isolated from the leg ulcer of 13 patients and 6 of these patients also harboured S. aureus in the nasal cavity. Klebsiella oxytoca was found in the ulcer and the nasal cavity of one patient. PFGE analysis revealed that patients harbouring $S$. aureus both in the nasal cavity and the leg ulcer had the same bacterial type at both sites. None of the S. aureus isolates were methicillin resistant.
\end{abstract}

Keywords: Staphylococcus aureus, nasal carriage, chronic leg ulcers, cross-contamination, Klebsiella oxytoca.

\section{INTRODUCTION}

Wound contaminants are likely to originate from three main sources: 1) the environment e.g. exogenous microorganisms in the air or those introduced by traumatic injury, 2) the surrounding skin and 3) endogenous sources involving mucous membranes [1]. Staphylococcus aureus is an important cause of human infections ranging from mild to lifethreatening and is frequently isolated from chronic leg ulcers [2]. S. aureus inhabit multiple sites of the skin and mucosal surfaces of carriers; however, the primary reservoir of staphylococci is believed to be the anterior nares. S. aureus nasal carriage is a major risk factor for both communityacquired and nosocomial infections and there seems to be an epidemiological link between nasal carriage and infections with S. aureus [3-7].

The purpose of this study was to investigate whether the bacterial species found in chronic leg ulcers were also present in the nasal cavity of the same patient.

Sixteen patients admitted to the Copenhagen Wound Healing Center, Bispebjerg Hospital, Denmark were included in the study. The patients suffered from persistent venous leg ulcers and all patients had previously been part of the SUE project [2]. A swab was taken from the ulcer and the nasal cavity of the patients. The swab was immediately placed in SSI Stuart transport medium (SSI 28733, Denmark) and processed the same day as collected.

Media and agar plates were purchased from Statens Serum Institut (SSI), Denmark. Swabs were cultured as described before [2]. All representative colonies were isolated and identified by standard microbiological methods, and where appropriate, commercial identification kits were used

*Address correspondence to this author at the Microbiology and Infection Control, Statens Serum Institut, Artillerivej 5, DK-2300 Copenhagen S, Denmark; Tel: +45 3268 3745; Fax: +45 3268 8238; E-mail: kak@ssi.dk to assist identification (VITEK GNI+, ID 32 Kits, BioMerieux, France).

All $S$. aureus strains found in this study and the SUE study [2] were tested. A bacterial suspension yielding semiconfluent growth was spread on a susceptibility $5 \%$ blood agar plate (SSI 723, Denmark) with the following NeoSensitabs (Rosco, Taastrup, Denmark): cefoxitin, penicillin, gentamicin, mecillinam, erythromycin, polymyxin, fucidin, vancomycin and rifampicin. Plates were incubated overnight at $37^{\circ} \mathrm{C}$, and inhibition zones were measured. Susceptibility interpretations were based on the manufacturer's recommendations.

All S. aureus strains were characterized by pulse-field gel electrophoresis (PFGE) after digestion with SmaI as described by Skov et al., 2005 [8].

S. aureus was found in 13 of the 16 ulcers. Six out of the 13 patients also harboured $S$. aureus in the nasal cavity. Klebsiella oxytoca was found in the ulcer and the nose of one patient, but besides this no other bacterial species isolated from the ulcers were found to be present in the nasal cavity. To the best of our knowledge, this is the first study on the possible transmission of $S$. aureus from the nasal cavity to chronic leg ulcers.

Fig. (1) shows the PFGE analysis of SmaI restriction fragments from patients harbouring $S$. aureus both in the nasal cavity and the leg ulcer. The figure reveals that these patients had the same type both places, thereby suggesting cross contamination between the nasal cavity and the leg ulcer. The PFGE type of the $S$. aureus isolates from the nasal cavity and leg ulcers of patient 4 and patient 5 was found to be identical. We cannot preclude that the strain had been transmitted from one patient to the other at the hospital premises. The result of the PFGE analysis is shown in Fig. (1). None of the $S$. aureus strains isolated from the chronic ulcers or the nasal cavity were found to be methicil- 
lin-resistant (MRSA). The strains isolated from the nasal cavity and the leg ulcers had identical resistance patterns. All isolates were sensitive to erythromycin, gentamicin, vancomycin and rifampicin and resistant to penicillin, mecillinam and polymyxin. Mecillinam and polymyxin are in general not considered to be functional against Gram positive bacterae. In one case the isolates from the nasal cavity and leg ulcer from the same patient were resistant to fucidin, while the remaining isolates were sensitive. These results are in accordance with the resistance profiles of 271 S. aureus isolates inhabiting chronic leg ulcer, which we also investigated (see Table 1). In this study we did not find any methicillin resistant $S$. aureus (MRSA). While MRSA is an increasing problem globally, the prevalence of MRSA in Denmark is quite low; in the period of 2005-2009 all departments of clinical microbiology in Denmark reported on S. aureus bacteraemia cases 6912 times; only $1.3 \%$ exhibited resistance to methicillin [9].

Bacterial species isolated from chronic ulcers were not found to be present in the nasal cavity except for the $S$. aureus strains and in one case; $K$. oxytoca. This indicates that the ulcer microbiota in general does not originate from the nasal cavity of the patients, with the exception of $S$. aureus. However, it is not possible to rule out the possibility that the nasal cavity might have been an intermediate reservoir at some time point for other bacterial species than $S$. aureus.

S. aureus was detected in the nasal cavity of $38 \%$ of the patients, moreover $S$. aureus were found in $81 \%$ of the leg ulcers identified using swab collections. We have recently shown that it is sufficient to use swab sampling to identify the bacterial species present in chronic wounds, thus avoiding complications associated with biopsy sampling [10]. The patients had the same $S$. aureus PFGE type in leg ulcers and nasal cavities, which indicates that cross-contamination between the ulcers and the nasal cavity occurs. Previous studies have shown that $S$. aureus originating in the nasal cavity may contaminate e.g. dialysis access sites $[5,6]$. Our study suggests that the source of contamination of leg ulcers may be the patients' anterior nares and further implicates that removal of infecting $S$. aureus from the leg ulcer may be very difficult if the reservoir (the nasal cavity) remains contaminated.

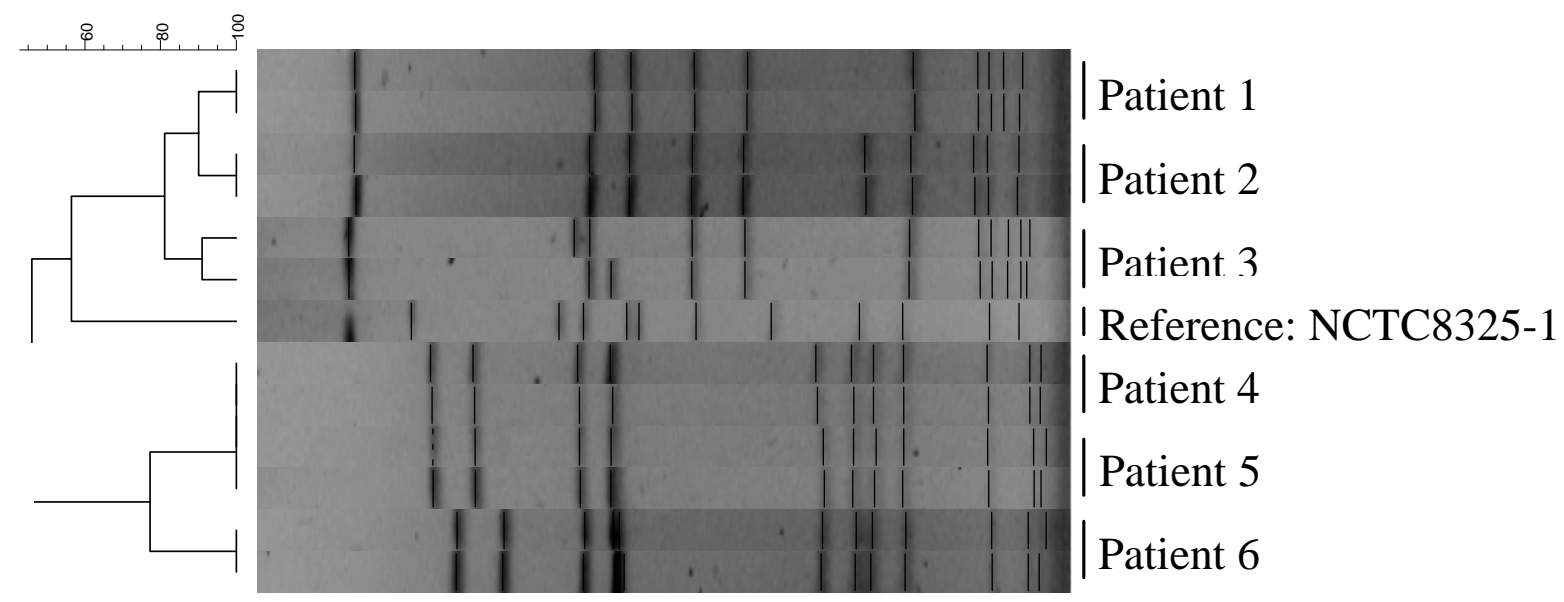

Fig. (1). PFGE of SmaI restriction fragments of S. aureus isolates. First lane for each patient is the isolate from the nasal cavity, second lane is from the leg ulcer.

Table 1. Antibiotic-resistance of 271 S. aureus Isolates from Chronic Venous Leg Ulcers

\begin{tabular}{|c|c|c|c|}
\hline Antibiotic & Resistant (\%) & Intermediary (\%) & Sensitive (\%) \\
\hline \hline Penicillin & $226(83.4)$ & $35(3.7)$ & $245(90.4)$ \\
\hline Erythromycin & $23(8.5)$ & $0(0.0)$ & $271(100.0)$ \\
\hline Gentamicin & $0(0.0)$ & $11(4.1)$ & $15(5.5)$ \\
\hline Mecillinam & $245(90.4)$ & $0(0.0)$ & $0(0.0)$ \\
\hline Polymyxin & $271(100.0)$ & $0(0.0)$ & $271(100.0)$ \\
\hline Cefoxitin & $0(0.0)$ & $44(16.2)$ & $201(74.2)$ \\
\hline Fucidin & $26(9.6)$ & $0(0.0)$ & $271(100.0)$ \\
\hline Vancomycin & $0(0.0)$ & $0(0.0)$ & $271(100.0)$ \\
\hline Rifampicin & $0(0.0)$ & & \\
\hline
\end{tabular}




\section{CONFLICT OF INTEREST}

The authors confirm that this article content has no conflicts of interest.

\section{ACKNOWLEDGEMENTS}

The study was partly financed by the Danish Research Agency under the SUE-programme (No. 9901187). Mette E. Skindersoe was supported by the Danish Centre for Antibiotic Research and Development (DanCARD, No. 09067075) financed by the Danish Council for Strategic Research.

\section{REFERENCES}

Bowler PG, Duerden BI, Armstrong DG. Wound microbiology and associated approaches to wound management. Clin Microbiol Rev 2001; 41: 244-69.

[2] Gjødsbøl K, Christensen JJ, Karlsmark T, Joergensen B, Klein BM, Krogfelt KA. Multiple bacterial species reside in chronic wounds: a longitudinal study. Int Wound J 2006; 3: 225-31.
[3] Luzar MA, Coles GA, Faller B, et al. Staphylococcus-aureus nasal carriage and infection in patients on continuous ambulatory peritoneal-dialysis. New Engl J Med 1990; 322: 505-9.

[4] Wenzel RP, Perl TM. The significance of nasal carriage of staphylococcus-aureus and the incidence of postoperative woundinfection. J Hosp Infec 1995; 31: 13-24.

[5] Yu VL, Goetz A, Wagener M, Smith PB, Rihs JD, Hanchett J, Zuravleff J. Staphylococcus-aureus Nasal carriage and infection in patients on hemodialysis - efficacy of antibiotic-prophylaxis. N Engl J Med 1986; 315: 91-6.

[6] Toshkova K, Annemuller C, Akineden O, Lammler C. The significance of nasal carriage of Staphylococcus aureus as risk factor for human skin infections. FEMS Microbiol Lett 2001; 202: 17-24.

[7] Eiff C von, Becker K, Machka K, Stammer H, Peters G. Nasal carriage as a source of Staphylococcus aureus bacteremia. N Engl J Med 2001; 344: 11-6.

[8] Skov R, Smyth R, Larsen AR, Frimodt-Moller N, Kahlmeter G. Evaluation of cefoxitin 5 and 10 microg discs for the detection of methicillin resistance in staphylococci. J Antimicrob Chemother 2005; 55: 157-61.

[9] Statens Serum Institut. Available at. http://www.danmap.org/pdfFiles/Danmap_2009.pdf. 2010.

[10] Gjodsbol K, Skindersoe ME, Christensen JJ, et al. No need for biopsies: comparison of three sample techniques for wound microbiota determination. Int Wound J 2012; 9: 295-302.

Received: October 09, 2012

Revised: November 26, 2012

Accepted: November 28, 2012

(C) Gjødsbøl et al.; Licensee Bentham Open.

This is an open access article licensed under the terms of the Creative Commons Attribution Non-Commercial License (http://creativecommons.org/licenses/ by-nc/3.0/) which permits unrestricted, non-commercial use, distribution and reproduction in any medium, provided the work is properly cited. 